\title{
Mercury Pump Using Rotating Permanent Magnets
}

\author{
Michael Butzek, and \\ Forschungszentrum Jülich, \\ Dr. I. Bucenieks \\ Institute of Physics Latvian University
}

The European Spallation Neutron Source (ESS) is the proposed next generation neutron source to be built in Europe. The two target stations, will be operated using liquid mercury as spallation target material and cooling fluid at the same time. During the spallation process 2.8 MW of the $5 \mathrm{MW}$ proton beam power is deposited in the mercury. In order to transport the heat to the facility raw water system, the mercury is pumped to a double wall heat exchanger acting on a intermediate water cooling loop. Due to the spallation process isotopes are formed within the mercury leading to radiation levels up to $500 \mathrm{~Sv} / \mathrm{h}$. In order to avoid spreading or mercury as well as radioactive products the mercury loop is operated inside a hot cell. A new type of MHD pump is proposed, using rotating permanent magnets. This pump represents a good compromise between mechanical pumps and EM-pumps under the given environmental conditions. This article will describe briefly the working principle of these pumps and discuss the benefit using this pump for ESS. In addition to that, a first design for this type of pump will be presented, taking into account ESS flow rate-, pressure-, handling- and environmental conditions. 